\title{
Article
}

Mycosphere

Doi 10.5943/mycosphere/7/4/2

Copyright (C) Guizhou Academy of Agricultural Sciences

\section{Characterization of four species including one new species of Agaricus subgenus Spissicaules from Eastern China}

\author{
Dai RC ${ }^{1,2}, \mathrm{Li} \mathrm{GJ}^{2}, \mathrm{He} M \mathrm{Q}^{1,2}, \mathrm{Liu} \mathrm{RL}^{3}$, Ling $\mathrm{ZL}^{2}, \mathrm{Wu} \mathrm{JR}^{1}$ and Zhao RL ${ }^{2}$ \\ ${ }^{1}$ Key Laboratory of Forest Disaster Warning and Control in Yunnan Province, Southwest Forestry University, \\ Kunming 650224, China. \\ ${ }^{2}$ State Key Laboratory of Mycology, Institute of Microbiology Chinese Academy of Sciences, Beijing \\ 100101, China. \\ ${ }^{3}$ Nature Reserve Administration Bureau of Zhejiang Province, Lishui City, Jingning County, Jingning 323500, \\ China.
}

Dai RC, Li GJ, He MQ, Liu RL, Ling ZL, Wu JR, Zhao RL 2016 - Characterization of four species including one new species of Agaricus subgenus Spissicaules from Eastern China. Mycosphere 7(4), 405-416, Doi 10.5943/mycosphere/7/4/2

\begin{abstract}
Four species of Agaricus subgenus Spissicaules, including a new species $A$. catenariocystidiosus, are identified from Eastern China based on morphological and molecular data. All of the species are introduced with full descriptions. Phylogenetic relationships were inferred based on the nuclear ribosomal internal transcribed spacer (ITS) region. The known species A. inthanoensis, A. parasubrutilescens and A. planipileus are compared with their original descriptions, and several supplementary morphological characters are provided.
\end{abstract}

Keywords - Agaricales - Agaricaceae - ITS - phylogeny - taxonomy

\section{Introduction}

Agaricus L. (Agaricales, Agaricaceae) is a genus that includes more than 400 species (Chen et al. 2012, 2015, Lebel et al. 2012, 2013, Li et al. 2014, Wang et al. 2015, Gui et al. 2015, Zhao et al. 2010, 2011, 2012, 2016). Edible mushrooms are generally low in calories and high in minerals, such as essential amino acids, vitamins and fibers (Mattila et al. 2002). The genus Agaricus contains many edible species, and some of them have been cultivated widely, such as A. bisporus (Large) Singer (Adams et al. 2008) and A. subrufescens Peck (Kerrigan 2005).

The epithet "Spissicaules" was first proposed for subsection Spissicaules Heimen. of section Agaricus based on the type species A. spissicaulis F.H. Møller (Heinemann 1978). Then, it was proposed and considered to be a section Spissicaules (Kerrigan 1985; Parra 2008) according to the possible affinities of A. lilaceps Heimen. Spissicaules contains A. lanipes (F.H. Møller \& Jul.Schäff.) Hlaváček, A. litoralis (Wakef. \& A. Pearson) Pilát and A. bresadolanus Bohus (Parra 2008). Species of section Spissicaules are characterized as follows: pileus diameter equal to, or smaller than, the length of the stipe; stipe often ventricose; cylindrical or clavate; mostly bulbous at the base; simple annulus; clearly striate on its upper surface; odor of anise or almond, sometimes very faint; $\mathrm{NaOH}$ reaction that is negative or very weak on all surfaces of the basidiome; and cheilocystidia clavate to cylindrical, sometimes septate at base, similar to immature basidia (Parra 2008). 
However, section Spissicaules has been revealed to be a possible polyphyletic group based on phylogenetic analyses using ITS sequences (Zhao et al. 2011). In the most recent systematic study on Agaricus using multigene sequences, subgenus Spissicaules (Heinem.) R.L. Zhao \& Moncalvo has been proposed to accommodate four sections: Amoeni Callac \& R.L. Zhao, Rarolentes Kerrigan, nom prov., Spissicaules (Heinem.) Kerrigan, and Subrutilescentes Kerrigan, nom. prov. (Zhao et al. 2016; Kerrigan 2016 in press).

We surveyed macrofungi from southwest of Zhejiang Province and north of Fujian Province, Eastern China, in 2015. Those areas have a subtropical monsoon climate, and their main vegetation is broad-leaved evergreen forests. The morphological and molecular phylogenetic studies of Agaricus specimens resulted in the discovery of one new species and three known species from subgenus Spissicaules.

\section{Materials \& Methods Morphological analyses}

Specimens were collected and photographed in the field. The macrocharacters were recorded when specimens were fresh, including the features of the pileus, lamella, stipe, annulus, odor and chemical reactions. Specimens were dried overnight in a food drier at $70^{\circ} \mathrm{C}$ after the morphological description was made. Dry specimens were kept in plastic bags, sealed and deposited in the Herbarium of Mycology, Institute of Microbiology, Chinese Academy of Sciences (HMAS). Microcharacters, such as basidia, basidiospores, cheilocystidia, pileipellis and annulus hyphae, were studied under a microscope (Olympus CX31). At least 20 measurements were made for each feature size for observations of basidiospores, basidia, cheilocystidia, pileipellis and annulus hyphae. Microscopic data were recorded as follows: $X=$ the mean of length by width $\pm \mathrm{SD}$; $\mathrm{Q}=$ the quotient of basidiospore length to width; and $\mathrm{Qm}=$ the mean of $\mathrm{Q}$ values $\pm \mathrm{SD} ; \mathrm{Q}=1.15-1.3$, broad ellipsoid; $\mathrm{Q}=1.3-1.6$, ellipsoid (Yang 2015). The description of morphological characters and chemical reactions followed Largent's methods (Largent 1986a, 1986b).

\section{Phylogenetic analyses}

The D3591-01 forensic kit (OMEGA Company, USA) was used for DNA extraction. The Taq PCR Mix (Beijing Biomed Company, China) was used for PCR amplification. Each amplification reaction volume was $50 \mu \mathrm{l}$ and contained the following: DNA template, $2 \mu \mathrm{l}$; PCR mix, $25 \mu \mathrm{l}$; ddH2O, $20 \mu \mathrm{l}$; and each primer, $1.5 \mu 1$. The primers used were ITS1 (5'GTAGGTGAACCTGCGG-3') and ITS4 (5'-TCCTCCGCTTATTGATATGC-3') (Li et al. 2012). The PCR cycling conditions were as follows: initial denaturation at $94{ }^{\circ} \mathrm{C}$, denaturation at $94{ }^{\circ} \mathrm{C}$ for $3 \mathrm{~min}$., annealing at $40^{\circ} \mathrm{C}$ for $30 \mathrm{~s}$, extension at $72{ }^{\circ} \mathrm{C}$ for $54 \mathrm{~s}$, for 35 cycles, with a final extension at $72{ }^{\circ} \mathrm{C}$ for $10 \mathrm{~min}$. The PCR amplicons were sent to Biomed Company for sequencing. Sequences were assembled in SeqMan (Swindel et al. 1997). The dataset used for phylogenetic analysis consisted of the ITS sequences produced from this study as well as additional sequences from GenBank. The dataset was initially aligned by ClustalX 2.0 (Thopmson et al. 1997) and then adjusted manually in BioEdit v. 7.0.4 (Hall 2007). The alignment was submitted to TreeBASE (submission ID 18397). The best substitution model was determined by MrModeltest 2.2 (Nylander 2004). The GTR+I+G nucleotide substitution model was used in the analysis and was detected by MrModeltest 2.2 (Nylander 2004). Maximum parsimony (MP) analyses was performed using PAUP*4.0b 10 (Swofford 2004), gaps in alignment were treated as missing data and the tree bisection-reconnection (TBR) algorithm was performed using the heuristic search option. Parsimony bootstrap values were obtained from 1000 bootstrap replicates. The consistency index (CI), retention index (RI), and tree length (TL) were calculated, and the max-trees were set to 1,000,000. MrBayes 3.2 (Ronquist et al. 2012) was used for the Bayesian analysis with four chains (one cold, three incrementally heated) for 10,000,00 generations, and trees were sampled every 100 generations. The procedure was stopped when the tree split deviation frequency value reached 0.01 , and the remaining trees were used to calculate the Bayesian posterior probabilities (PP) of individual clades. 


\section{Results}

MP and Bayesian tree topologies were the same, so the Bayesian tree was used for the results. The dataset contained 79 ITS sequences, of which 17 were produced from this study. The subgenus Spissicaules includes 4 clades with good support: sect. Surbrutilescentes (clade A) with moderate support $(\mathrm{PP} / \mathrm{BS}=0.63 / 72)$, sect. Amoeni $($ clade $\mathrm{B})$ with strong support $(\mathrm{PP} / \mathrm{BS}=$ $0.94 / 61$ ), sect. Rarolentes (clade C) with full support $(\mathrm{PP} / \mathrm{BS}=1 / 100)$ and sect. Spissicaules (clade D) with moderate support $(\mathrm{PP} / \mathrm{BS}=0.6 / 100)$. In the phylogenetic tree (Fig. 1), specimens ZRL2015038, ZRL20151199 and ZRL20151210 composed a separate clade with full support (PP/BS $=1 / 100)$ in Clade A (sect. Subrutilescentes). Specimens ZRL2015060, ZRL20150110, ZRL2015106 and ZRL2015112 nested in the clade of A. parasubrutilescens Callac and R.L. Zhao with a moderately supported PP/BS value of 0.73/78. Specimens ZRL20151566, ZRL2015166 belong to a branch of A. inthanoensis L.J. Chen, K.D. Hyde \& R.L. Zhao and A. brunneopileatus Callac \& R.L. Zhao and have strongly supported PP/BS values (0.99/72). Specimens ZRL20151230, ZRL20151244, ZRL20151241, ZRL20151565, ZRL20151193, ZRL20151574, ZRL20151239 and ZRL20151222 join the clade of A. planipileus R.L. Zhao with strong support $(\mathrm{PP} / \mathrm{BS}=1 / 88)$.

\section{Taxonomy}

Agaricus catenariocystidiosus R.C. Dai \& R.L. Zhao, sp. nov.

Fig. 2

Etymology - refers to the cheilocystidia are in chains.

Typus - China, Zhejiang Province, Lishui City, Jingning County, Caoyutang Forest Park, N 275' E 119³8', elv.1200 m., 2nd June 2015, Zhao Rui-Lin, ZRL2015038 (HMAS 280112)

Diagnosis - the morphological characters that distinguish A. catenariocystidiosus from other Agaricus species are its chain-like cheilocystidia, longer basidiospores, $\mathrm{NaOH}$ reaction turns weakly green, odor of phenol.

Description - Pileus 42-98 $\mathrm{mm}$ in diam., convex and umbonate, cap margin entire, surface dry, covered with appressed, triangular fibrillose squamules, dense at disc, brown to light brown towards the margin, background dirty white. Context 1-6 mm thick, white and flesh. Lamellae free, crowded, intercalated with lamellulae; 3-5 mm broad; at first white, then grayish white, reddish brown, brown, finally dark brown. Stipe apex 4-7 mm, base 6-23 $\mathrm{mm}$ in diameter, 68-96 $\mathrm{mm}$ in length, cylindrical to long clavate, hollow, surface smooth above the ring, fibrillose scales under the ring, white. Annulus single, membranous, superous, edge entire, white, upside smooth, lower side floccose. Odor of phenol. Discoloration indistinct on touching and cutting, or turning brown on context exposure after 5 mins.

Macrochemical reaction $-10 \% \mathrm{NaOH}$ reaction weakly green on surface.

Basidiospores 5-6 × 3-4 $\mu \mathrm{m}[\mathrm{x}=5.2 \pm 0.3 \times 3.4 \pm 0.1, \mathrm{Q}=1.3-1.8, \mathrm{Qm}=1.5 \pm 0.1, \mathrm{n}=20]$, ellipsoid, no germ pore, smooth, brown. Basidia 15-24 × 6-9 $\mu \mathrm{m}$, hyaline, smooth, cylindrical or clavate, 4-spored. Cheilocystidia 13-28 $\times$ 8-18 $\mu \mathrm{m}$, smooth, mostly pyriform, clavate, spherical, some in chains, hyaline. Pleurocystidia absent. Pileipellis cutis composed of hyphae 3-16 $\mu \mathrm{m}$ in width, constricted at the septa, contains brown membranous pigments. Annulus composed of hyphae 4-10 $\mu \mathrm{m}$ in width, smooth, clavate, hyaline.

Habit - solitary, scattered or gregarious on soil of broad-leaved forests.

Other specimens examined - China, Zhejiang Province, Lishui City, Jingning County, Baiyun, Wangdongyang Natural Reserve, N 2758' E 119³8', elv. 1200 m., 11 August 2015, Zhao Rui-Lin, ZRL20151210 (HMAS 275805); ibid, He Mao-Qiang, ZRL20151199 (HMAS 275804).

Notes - The morphological characters of A. catenariocystidiasus match the circumscription of section Subrutilescentes well (Zhao et al. 2016), and the phylogenetic analysis also supports its placement in this section. Compared with the known species of section Subrutilescentes and section Spissicaules, Agaricus catenariocystidiasus is different from A. Linzhiensis and A. brunneopileatus by having wider or thinner basidiospores than A. catenariocystidiasus in Zhao et al. (2016), A. Linzhiensis spores that are broadly ellipsoid, $\mathrm{Qm}=1.3 ;$ A. brunneopileatus spores elongate ellipsoid 


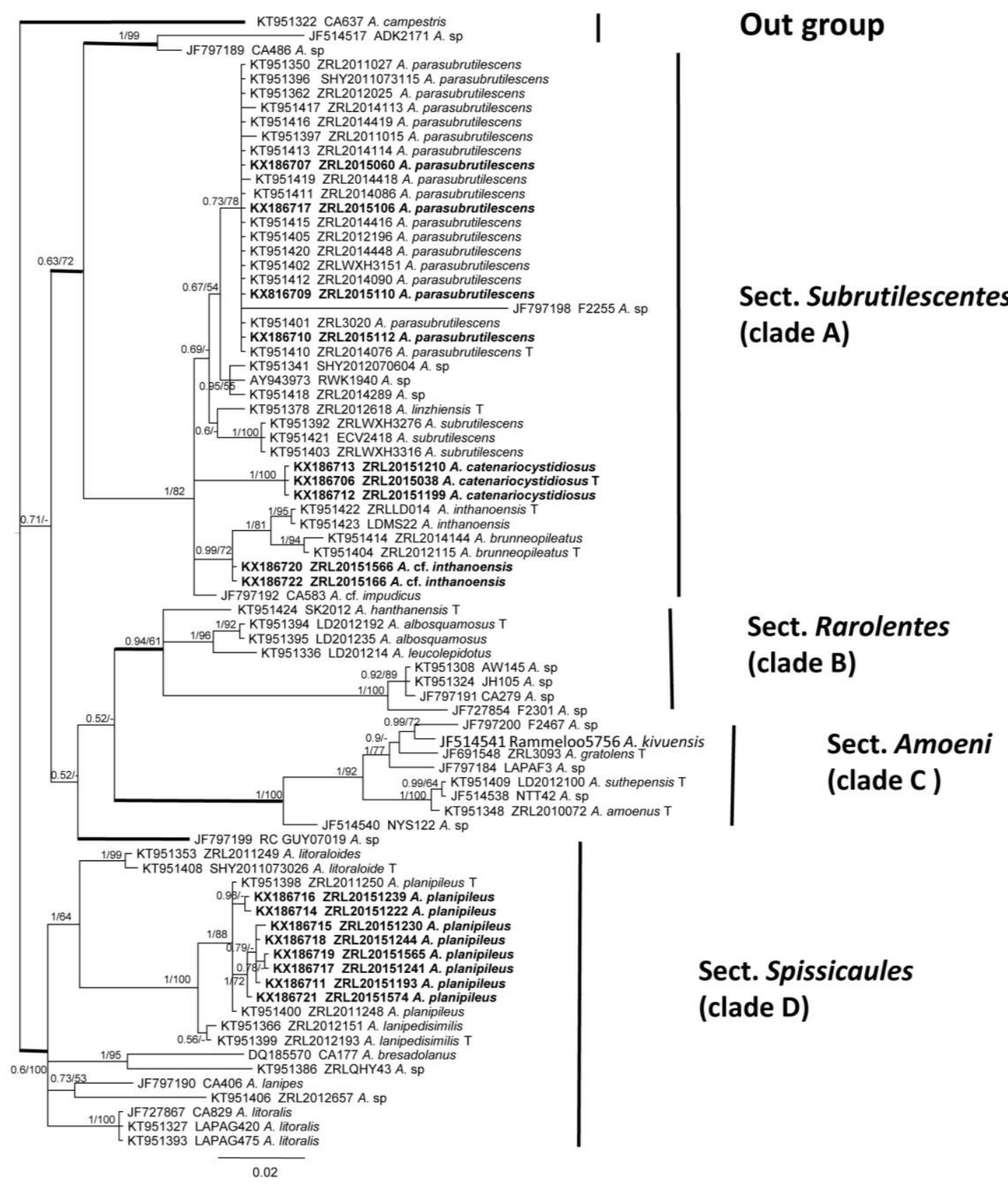

Fig. 1 - Phylogeny of Agaricus subgenus Spissicaules generated from the Bayesian analysis of the ITS sequences. Bayesian posterior probability (PP) values and Bootstrap support (BS) values $>50 \%$ are given at the internodes (PP/BS). Agaricus campestris (CA637), A. sp (ADK2171) and $A$. sp (CA486) were used as an outgroup. T indicates the type species. Sequences produced from this study are in bold. Four sections are indicated.

Qm =1.7). Agaricus impudicus develops strong red or reddish brown discoloration on its gills when bruised, while A. catenariocystidiosus lacks such a discoloration. Agaricus planipileus and A. inthanonensis differ by developing a distinct yellow discoloration upon cutting. Comparing with $A$. parasubrutilescens, A. catenariocystidiasus has chain-like cheilocystidia and a $10 \% \mathrm{NaOH}$ reaction leads it to be weakly green on the surface. However, in A. parasubrutilescens, its cheilocystidia are pyriform without in chains and the $10 \% \mathrm{NaOH}$ reaction is indistinct.

The most similar species to A. catenariocystidiasus in morphology is A. subrutilescens because both have a greenish discoloration when reacted with $\mathrm{NaOH}$, which is rare within Agaricus species (Kerrigan, 1986). However, the latter species differs by its dark brown, reddish brown scales on the pileus and simple cheilocystidia. The phylogenetic analysis also supports them as different species. 

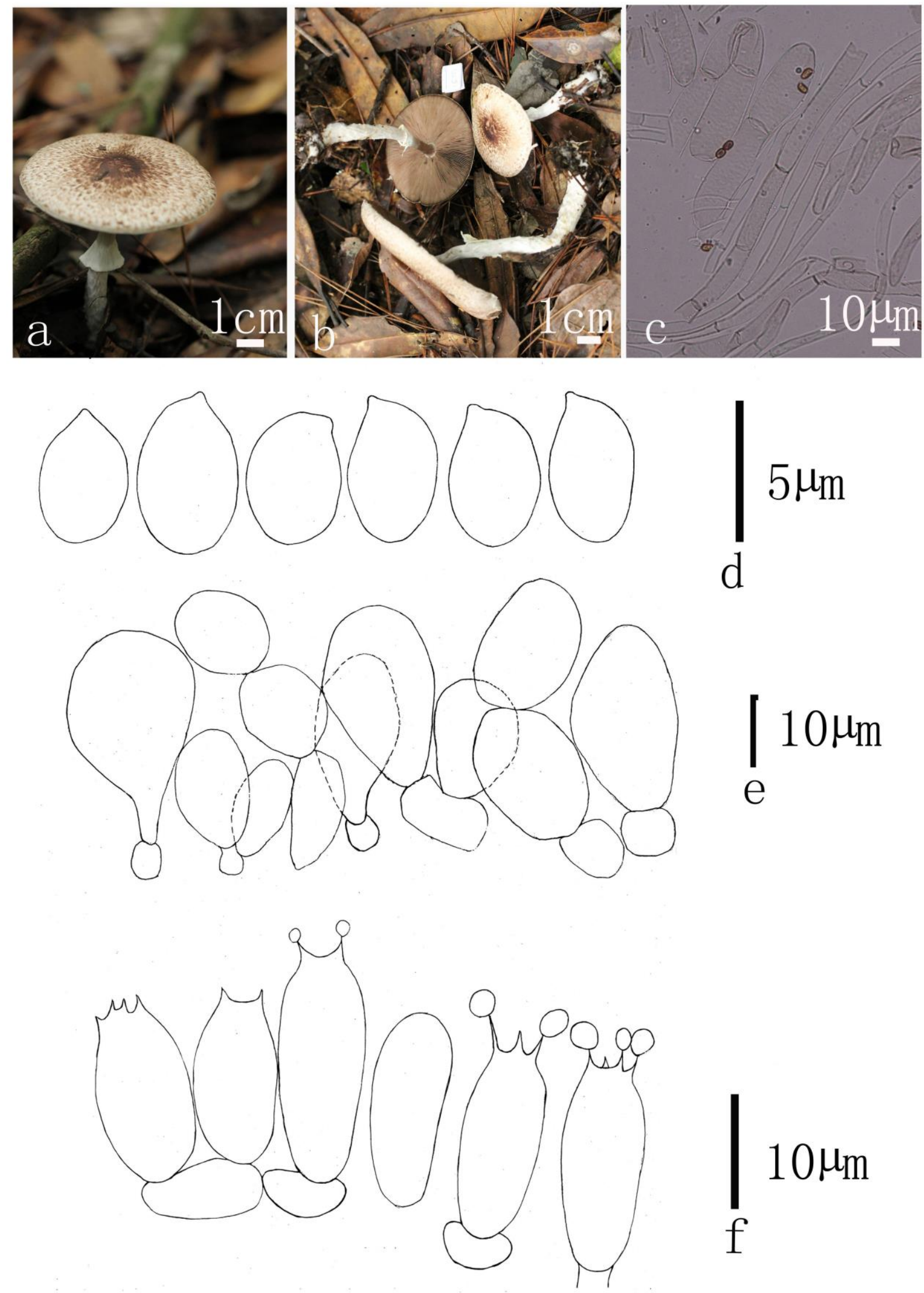

Fig 2. - Agaricus catenariocystidiosus: ZRL2015038 (HMAS 280112 type) a-b. Basidiomata in field; c. Pileipellis hyphae; d. Basidiospores; e. Cheilocystidia; f. Basidia. 
Description - Pileus 76-91 $\mathrm{mm}$ in diam., plane and slightly depressed, cap margin entire, surface dry, covered with appressed, triangular fibrillose-scales, brown scales on a light brown background. Context 5-6 mm thick, flesh. Lamellae free, crowded, intercalated with lamellulae, 6-8 $\mathrm{mm}$ broad, brown. Stipe apex $8 \mathrm{~mm}$, base 11-21 $\mathrm{mm}$ in diameter, length 73-109 mm, cylindrical, base bulbous, hollow, surface smooth above the ring, fibrillose scales below the ring, white. Annulus single, membranous, superous, edge entire, persistent, white, upside smooth, lower side floccose with brown pigments. Odor of phenol. Discoloration light yellow on bruising at the surface of pileus.

Macrochemical reaction $-10 \% \mathrm{NaOH}$ reaction yellow on surfaces of stipe and cap.

Basidiospores 5-7 × 3-5 $\mu \mathrm{m}[\mathrm{x}=6.0 \pm 0.4 \times 4.0 \pm 0.3, \mathrm{Q}=1.3-1.7, \mathrm{Qm}=1.5 \pm 0.1, \mathrm{n}=$ 20], ellipsoid, no germ pore, smooth, brown. Basidia 14-26 × 5-8 $\mu \mathrm{m}$, hyaline, smooth, cylindrical or clavate, 4-spored. Cheilocystidia 9-17 × 6-13 $\mu \mathrm{m}$, smooth, ellipsoid, clavate or spherical. Pleurocystidia absent. Pileipellis cutis composed of hyphae $4-11 \mu \mathrm{m}$ in width, constricted at the septa, contains brown membranous pigments. Annulus composed of hyphae 3-11 $\mu \mathrm{m}$ in width, smooth, clavate and hyaline.

Habit - solitary, scattered or gregarious on soil of broad-leaved forests.

Specimens examined - China, Fujian Province, Wuyi Mountains Natural Nationsal

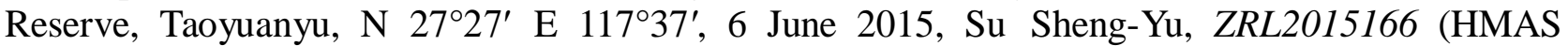
275816). China, Zhejiang Province, Jingning, Caoyutang Forest Park, N 2758' E 119 $38^{\prime}$, elv.1200 m.,19 August 2015, Ling Zhi-Lin, ZRL20151566 (HMAS

275814). Thailand, Chiang Mai Province, Doi Inthanon, Sit 1, N 1906'28.8" E98 44'47.3", elv. 1050 m., 1 July 2010, Chen Jie, LD014 (MFLU 10-0747 Holotype).

Notes - The morphological characters of the specimens from this study generally match those of A. inthanoensis (Zhao et al. 2016), with the exception of the $10 \% \mathrm{NaOH}$ reaction, which is absent from the original description, but is yellow in the specimens from this study. However, in the phylogenetic tree, they are separated. Currently, we have named these specimens as $A$. cf. inthanonensis. Information from multigene sequence analyses may be needed for a more precise identification.

Agaricus planipileus R.L. Zhao, Fungal Divers. 2016 in press, Fig. 4

Description - Pileus 52-120 $\mathrm{mm}$ in diam., parabolic to convex with truncate disc when young, then convex to plane with a slight depressed disc in age, margin entire, smooth or appendiculate, surface dry, covered with fibrillose-scales in whole surface, triangular shape or not, brown. Lamellae free, crowded, intercalated with lamelullae, 8.4-10 mm broad, white or little pink, grayish white, to dark brown in age. Context 5-9 mm thick, flesh, white. Stipe apex 8-13 $\mathrm{mm}$, base 18-33 $\mathrm{mm}$ in diameter, length 69-128 $\mathrm{mm}$, clavate-bulbous, hollow, surface smooth above ring, floccose to heavily squamose below ring, white, some squames with brown tips. Annulus $10 \mathrm{~mm}$ diam., single, membranous, superous, white, upside smooth, lower side floccose, white or light brown fibrillose. Odor of almonds or aniseed. Discoloring on surfaces of pileus and stipe on bruising, handling or scratching; discoloring light orange on context exposure.

Macrochemical reaction $-10 \% \mathrm{NaOH}$ reaction colored the stipe and cap yellow.

Basidiospores 4-7 × 3-5 $\mu \mathrm{m}[\mathrm{x}=5.3 \pm 0.5 \times 3.6 \pm 0.5, \mathrm{Q}=1.3-1.7, \mathrm{Qm}=1.5 \pm 0.2, \mathrm{n}=20]$, ellipsoid or broad ellipsoid, no germ pore, smooth, brown. Basidia 14-23 × 6-9 $\mu \mathrm{m}$, hyaline, smooth, clavate to broadly clavate, 4-spored. Cheilocystidia 9-29 ×9-17 $\mu \mathrm{m}$, smooth and hyaline, elliptic, spherical, pyriform, clavate, few separated, some specimens lacking or basidia-liked. Pleurocystidia absent. Pileipellis cutis composed of hyphae 3-14 $\mu \mathrm{m}$ in width, constricted at the septa, contains brown membranous pigments.

Habit: solitary or in troops of broad-leaved forest.

Specimens examined - China, Zhejiang Province, Lishui City, Jingning County, Baiyun, Wangdongyang Natural Reserve. N 2758' E 119³8', elv.1200 m., 11 August 2015, Zhou Jie- 

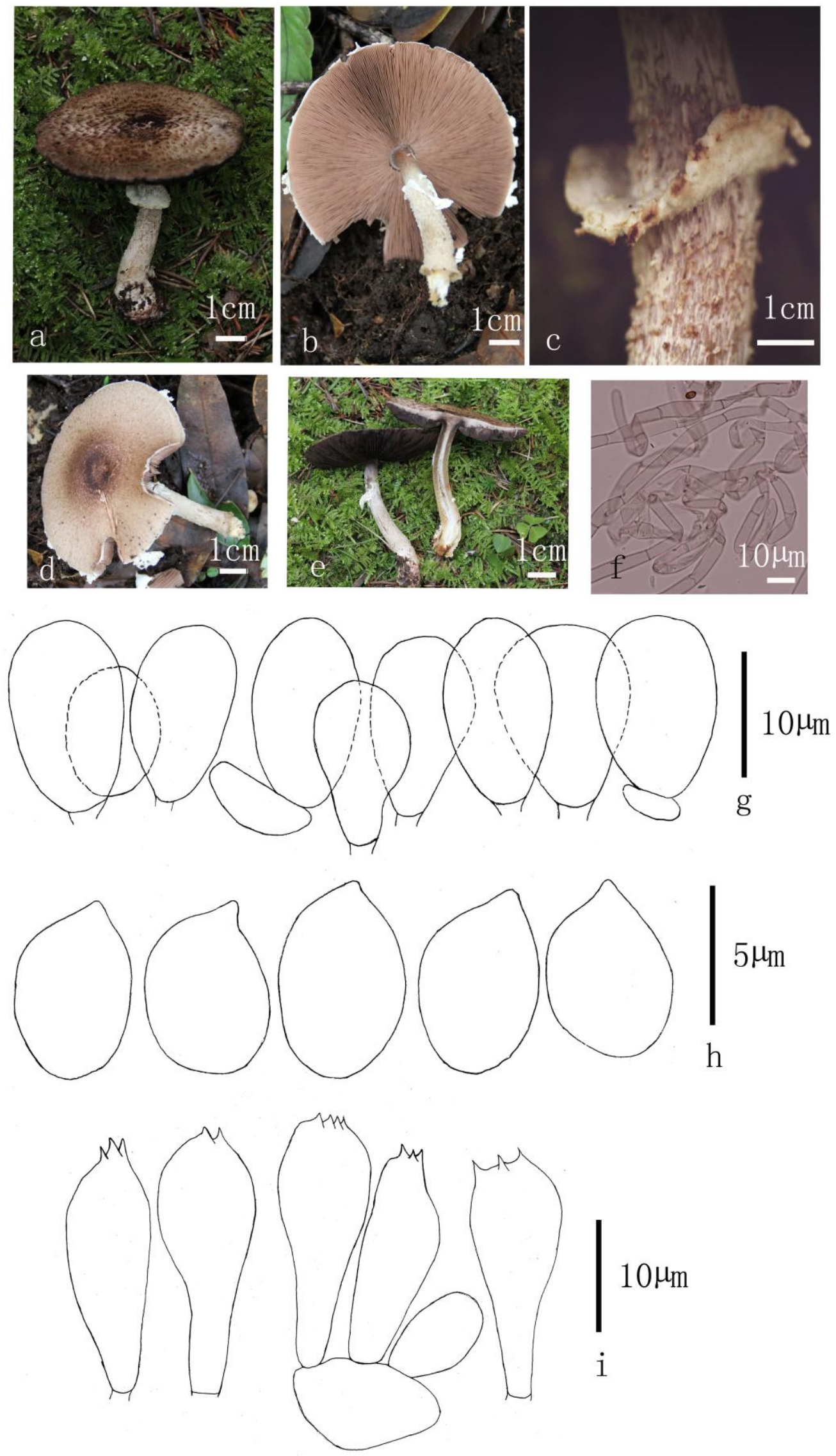

Fig. 3 - Agaricus cf. inthanonensis: a, c, e from specimen ZRL20151566 (HMAS 275814); b, d, fi from ZRL2015166 (HMAS 275816); a-e Basidiomates in the field; f. Pileipellis hyphae; g. Cheilocystidia; h. Basidiospores; i. Basidia. 

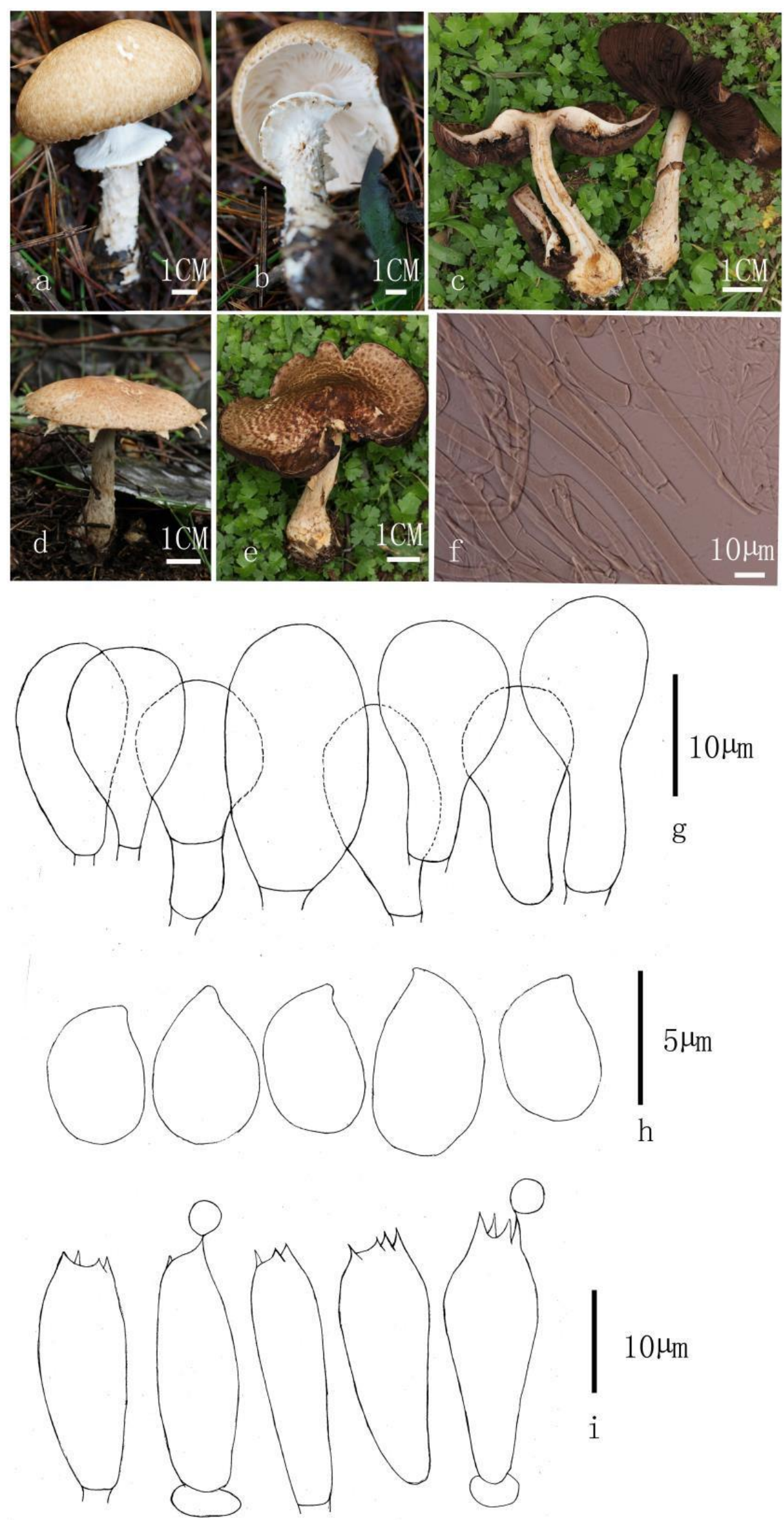

Fig. 4 - Agaricus planipileus: specimen a, b, f-i from ZRL20151193 (HMAS 275808); c, e from ZRL20151565 (HMAS 280114); d from ZRL20151239 (HMAS 275809); f. Pileipellis hyphae; g. Cheilocystidia; h. Basidiospores; i. Basidia. 

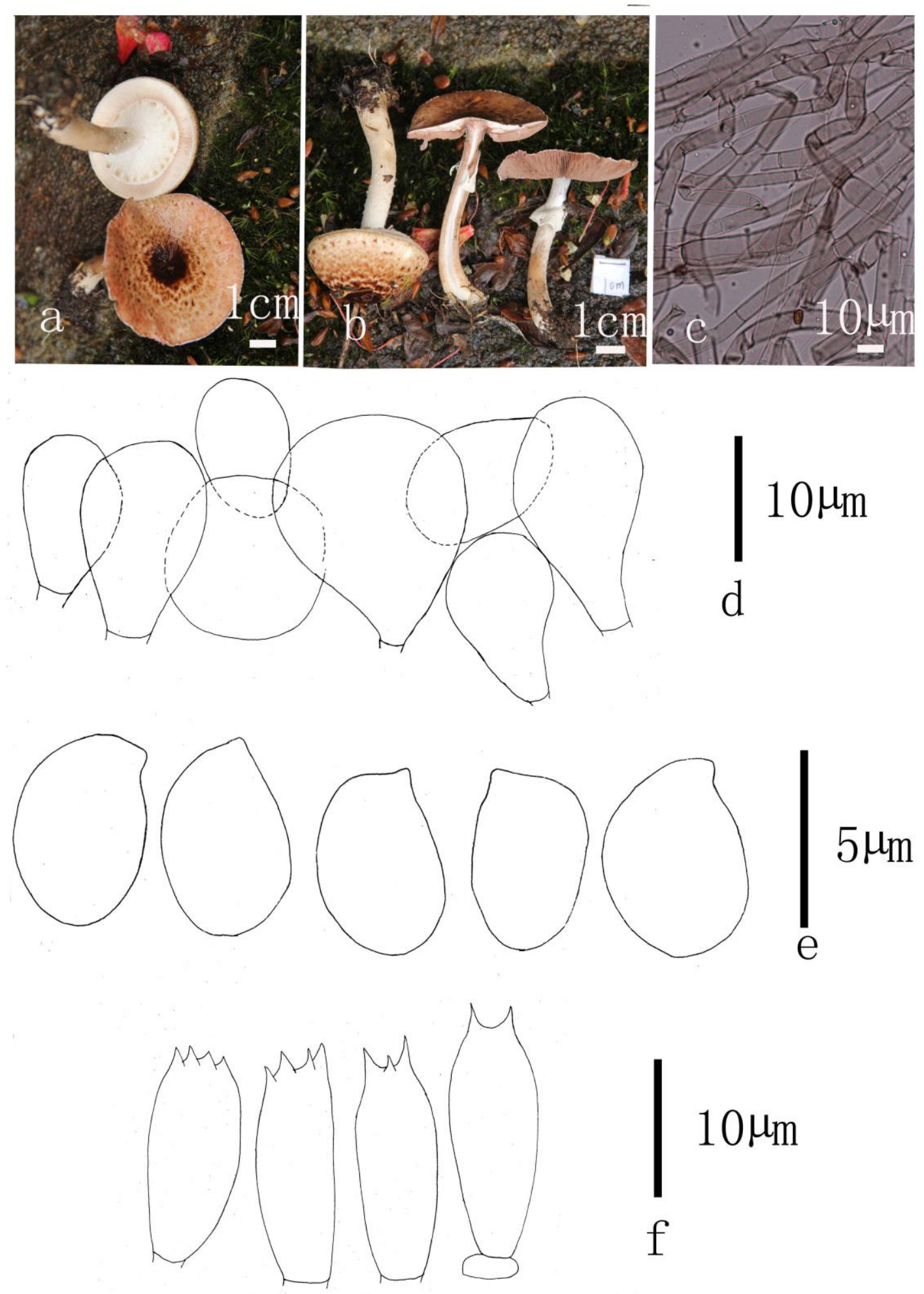

Fig. 5 - Agaricus parasubrutilescens: a-f from ZRL2015112 (HMAS 275811); c. Pileipellis hyphae; d. Cheilocystidia; e. Basidiospores; f. Basidia.

Min, ZRL20151193 (HMAS 275808); ibid, He Mao-Qiang, ZRL20151241 (HMAS 280113); ibid, Zhao Rui-Lin ZRL20151222 (HMAS 280115); ibid, Zhao Rui-Lin, ZRL20151239 (HMAS 275809); ibid, ZRL20151244 (HMAS 275807); ibid, Huangtian Lake, 20 August 2015, He MaoQiang, ZRL20151574 (HMAS 275806); ibid, Caoyutang, Forest park Natural Reserve, 19 August 2015, He Mao-Qiang, ZRL20151565 (HMAS 280114). China, Yunnan Province, Tengchong county, Datang, Danlonghe Village, 28 July 2011, Zhao Rui-Lin, ZRL2011250 (HMAS 273997 Holotype). 
Notes - The morphological characters of the specimens in this study match the original description (Zhao et al. 2016), and the phylogenetic analyses also support their classification as the same species under the name $A$. planipileus. The species has additional $1 \mathrm{bp}$ in ITS sequences than originally reported. In the original description, the $10 \% \mathrm{NaOH}$ reaction was not detected, but from the examination of our specimens, the $\mathrm{NaOH}$ reaction is yellow, which would be a supplementary character for this species definition. The specimens ZRL20151239 and ZRL20151222, are lacking cheilocystidia, maybe the specimens are too young or too old.

Agaricus parasubrutilescens Callac \& R.L. Zhao, Fungal divers. 2016 in press

Fig. 5

Description - Pileus 22-81 $\mathrm{mm}$ in diam., convex to applanate, cap margin striate, surface dry, covered with triangular fibrillose-scales, brown scales on light brown background. Context 4-5 mm thick, flesh, white. Lamellae free, crowded, intercalated in lamelullae, 3-4 mm broad, pink when young, then brown, dark brown when old, edges even. Stipe apex 7-9 mm, base 14-17 $\mathrm{mm}$ in diameter, length $64-102 \mathrm{~mm}$, clavate, base bulbous, hollow, surface smooth, white above ring, fibrillose scales white and light brown towards base below ring. Annulus single, membranous, superous, upside smooth and

white, lower side floccose. Odor is aniseed. No discoloration on touching; discoloring reddish brown on context exposure of stipe.

Macrochemical reaction $-10 \% \mathrm{NaOH}$ reaction is indistinct.

Basidiospores 4-6 × 3-4 $\mu \mathrm{m}[\mathrm{x}=4.7 \pm 0.4 \times 3.2 \pm 0.3, \mathrm{Q}=1.2-1.8, \mathrm{Qm}=1.5 \pm 0.2, \mathrm{n}=20]$, ellipsoid or broad ellipsoid, no germ pore, smooth, brown. Basidia 14-18 $\times 5-7 \mu \mathrm{m}$, hyaline and smooth, cylindrical, 4-spored. Cheilocystidia 11-19 × 7-16 $\mu \mathrm{m}$, smooth and hyaline, some pyriform or clavate, mostly elliptic and spherical. Pleurocystidia absent. Pileipellis cutis, composed of hyphae 3-12 $\mu \mathrm{m}$ in width, constricted at the septa, contains brown membranous pigments. Annulus composed of hyphae 4-9 $\mu \mathrm{m}$ in width, smooth, clavate and hyaline.

Habit - solitary, scattered or gregarious on soil in forest.

Specimens examined - China, Zhejiang Province, Lishui City, Jingning County, Baiyun, Wangdongyang Natural Reserve, N 2758’ E 119³8', elv.1200 m., 4 June 2015, Su Sheng-Yu, ZRL2015112 (HMAS 275811); ibid, ZRL2015110 (HMAS 275812); ibid, He Mao-Qiang, ZRL2015106 (HMAS 275813); ibid, Caoyutang, Forest Park, 2 June 2015, He Mao-Qiang, ZRL2015060 (HMAS 275810). China, Yunnan Province, Dali County, Cangshan, 24 July 2014, Su Sheng-Yu, ZRL2014076 (HMAS274000 Holotype).

Notes - the morphological and phylogenetic analyses support the classification of the specimens in this study as the known species A. parasubrutilescens (Zhao et al. 2016). This species is widely distributed in tropical and subtropical areas, such as Thailand and southern China (Yunnan and Guangdong Provinces) (Zhao et al. 2016). The discovery of this species in Zhejiang Province is the most northern distribution described to date.

\section{Acknowledgements}

Su Sheng-Yu and Zhou Jie-Min are thanked for their help in collecting specimens. This work is financed by the National Natural Science Foundation of China [31000013, 31360014, 31470152, 31500013] and the support of developing subject.

\section{References}

Adams LS, Phung S, Wu X, Ki L, Chen S. 2008 - White button mushroom (Agaricus bisporus) exhibits antiproliferative and proapoptoties and inhibits prostate tumor growth in athymic mice. Nutrition and Cancer, 60 (6): 744-756.

Chen J, Zhao RL. Karunarathna SC, Callac P, Raspé O, Bahkali AH, Hyde KD. 2012. - Agaricus megalosporus: a new species in section Minores. Cryptogamie, Mycologie, 33: 145-155.

Chen J, Zhao R, Parra LA, Guelly AK, De Kesel A, Rapior S, Hyde KD, Chukeatirote E, Callac P. 2015. - Agaricus section Brunneopicti: a phylogenetic reconstruction with descriptions of 
four new taxa. Phytotaxa, 192 (3): 145-168.

Gui Y, Zhu GS, Callac P, Hyde KD, Parra LA, Chen J, Yang TJ, Huang WB, Gong GL, Liu ZY. 2015. - Agaricus section Arvenses: three new species in highland subtropical Southwest China. Fungal biology, 119: 79-94.

Heinemann P. 1978. - Essai d'une clédune clé de determination des genres Agaricus et Micropsalliota. Sydowia 30: 6-37.

Hall T. 2007. - BioEdit v7. Available from: http: //www. mbio. ncsu. edu/BioEdit/BioEdit. html.

Kerrigan RW. 1985. - Studies in Agaricus II. Agaricus liaceps re-evaluated. Mycologia 77 (1): $137-141$.

Kerrigan RW. 1986. - Agaricales of California. Vol. 6. Agaricaceae. Mad River Press, Eureka, $1-62$.

Kerrigan RW. 2005. - Agaricus subrufescens, a cultivated edible and medicinal mushroom, and its synonyms. Mycologia 97: 12-24.

Kerrigan RW. 2016. - Agaricus of North America. New York Botanical Garden Press, Bronx, (in press)

Largent DL 1986a. - How to identify mushrooms to genus vol. 1. Macroscopic features. Mad River Press, Eureka, p 166.

Largent DL 1986b. - How to identify mushrooms to genus vol. 3. Microscopic features. Mad River Press, Eureka, p 148.

Lebel T, Syme A. 2012. - Sequestrate species of Agaricus and Macrolepiota from Australia: new species and combinations and their position in a calibrated phylogeny. Mycologia, 104: 496-520.

Lebel T. 2013. - Two new species of sequestrate Agaricus (section Minores) from Australia. Mycological progress, 12: 699-707.

Li GJ, Li SF, Liu XZ, Wen HA. 2012. - Russula jilinensis sp. nov. (Russulaceae) from northeast China. Mycotaxon, 120: 49-58.

Li SF, Xi YL, Qi CX, Liang QQ, Wei SL, Li GJ, Zhao D, Li SJ, Wen HA. 2014. - Agaricus taeniatus sp. nov. a new member of Agaricus sect. Bivelares from northwest China. Mycotaxon, 129: 187-196.

Mattila P, Salo-Väänänen P, Könkö K, Aro H, Jalava T. 2002. - Basic composition and amino acid contents of mushrooms cultivated in Finland. Journal of Agricultural \& Food Chemistry, 50 (22): 6419-6422.

Nylander JAA 2004. - MrModeltest 2.2. Program distributed by the author. Uppsala: Uppsala University, Uppsala: Department of Systematic Zoology, Evolutionary Biology Centre.

Parra LA. 2008. - Agaricus L Allopsalliota Nauta \& Bas. Alassio: Edizioni Candusso. Ronquist F, Teslenko M, Mark P, Ayres DL, Darling A, Höhna S, Larget B, Liu L,

Suchard MA, Huelsenbeck JP. 2012. - MrBayes 3.2: Efficient Bayesian Phylogenetic Inference and Model Choice Across a Large Model Space Biology. 61 (3): 539-542.

Swindell SR, Plasterer TN. 1997. - SEQMAN. Sequence data analysis guidebook, 75-89. Swofford DL. 2004. - PAUP*: phylogenetic analysis using parsimony, Version 4.0b10. Sunderland: Sinauer.

Thopmson JD, Gibson TJ, Plewniak F, Jeanmougin F, Higgins DG. 1997. - The Clustal_X windows interface: flexible strategies formultiple sequence alignment aided by quality analysis tools. Nucleic acids research. 25 (24): 4876-4882.

Wang ZR, Parra LA, Callac P, Zhou JL, Fu WJ, Dui SH, Hyde KD, Zhao RL. 2015. - Edible species of Agaricus (Agaricaceae) from Xinjiang Province (Western China). Phytotaxa. 202 (3): 185-197.

Yang ZL. 2005. - Flora fungorum sinicorum, vol 27 Amanitaceae (in Chinese). Science Press, Beijing.

Zhao RL, Desjardin DE, Soytong K, Perry BA, Hyde KD. 2010. - A monograph of Micropsalliota in Northern Thailand based on morphological and molecular data. Fungal Diversity. 45 (1): 33-79. 
Zhao RL, Karunarathna SC, Raspé O, Parra LA, Guinberteau J, Moinard M, De Kesel A, Barroso G, Courtecuisse R, Guelly AK, Desjardin DE, Hyde KD, Callac P. 2011. - Major clades in tropical Agaricus. Fungal Diversity. 51: 279-296.

Zhao RL, Hyde KD, Karunarathna SC, Desjardin DE, Raspé O, Soytong K, Guinberteau J, Callac P. 2012. - Agaricus flocculosipes sp. nov., a new potentially cultivatable species from the palaeotropics. Mycoscience. 53 (4): 300-311.

Zhao RL, Zhou JL, Chen J, Margaritescu S, Sánchez-Ramírez S, Hyde KD, Callac P, Parra LA, Li GJ, Moncalvo JM. 2016. -Towards standardizing taxonomic ranks using divergence times - a case study for reconstruction of the Agaricus taxonomic system. Fungal Diversity, 78 (1): 239-292. 